Pacific Journal of Mathematics

A NUMERICAL CONDITION FOR MODULARITY OF A

s. 


\section{A NUMERICAL CONDITION FOR MODULARITY OF A LATTICE}

S. P. Avann

1. Introduction. In this note a simple numerical condition $(\theta)$ is presented which is necessary for modularity of a finite lattice $L$. Though not sufficient $(\theta)$ appears to be a condition imposing a strong tendency toward modularity.

Notation. Covering, proper inclusion, and inclusion will be denoted by $>, \supset$, $\supseteqq$ respectively. $N[S]$ will denote the order of the set $S$. The unit and zero elements will be denoted by $u$ and $z$ respectively.

Definition 1. A finite lattice $L$ is upper semi-modular [1: p. 100] if and only if

$$
a \text { and } b>a \cap b \text { imply } a \cup b>a \text { and } b \text {. }
$$

$L$ is lower semi-modular if and only if

$$
a \cup b>a \text { and } b \text { imply } a \text { and } b>a \cap b \text {. }
$$

Definition 2. In a finite lattice let $C(a)=\{x \in L \mid x<x \cup a>a\}$ and $D(a)=\{x \in L \mid x>x \cap a<a\}$.

2. Tests for modularity An immediate consequence of Definitions 1 and 2 is the following theorem.

Theorem 1. In a finite lattice $L$ condition $\left(\xi^{\prime}\right)$ is equivalent to $D(a)$ $\leqq C(a)$ for all $a \in L$ and both imply $N[D(a)] \leqq N[C(a)]$. Dually, $\left(\xi^{\prime \prime}\right)$ is equivalent to $D(a) \supseteqq C(a)$ for all $a \in L$ and both imply $N[D(a)] \geqq N[C(a)]$. Moreover, modularity, $\left(\xi^{\prime}\right)$ and $\left(\xi^{\prime \prime}\right)$, is equivalent to $D(a)=C(a)$ for all $a \in L$ and both imply the condition $(\theta)$ :

$$
N[D(a)]=N[C(\alpha)] \text { for all } a \in L \text {. }
$$

The contrapositive of the last statement of Theorem 1 serves as a useful test for non-modularity :

THEOREM 2. If there exists $a \in L$ for which $N[D(a)] \neq N[U(a)]$, then $L$ is non-modular.

When either $\left(\xi^{\prime}\right)$ or $\left(\xi^{\prime \prime}\right)$ is known to hold in $L$, the verification of the condition $(\theta)$ is a test often easiest to apply. It merely requires counting coverings. 
THEOREM 3. In a finite lattice $L\left(\xi^{\prime}\right)$ and $(\theta)$ together imply modularity and dually $\left(\xi^{\prime \prime}\right)$ and $(\theta)$ together likewise imply modularity.

Proof. From Theorem 1 condition $\left(\xi^{\prime}\right)$ implies $D(a) \leqq C(a)$, and along with $(\theta)$ we obtain $D(a)=C(a)$ for all $a \in L$. Hence $L$ is modular.

Condition $(\theta)$ appears to be a very strong condition toward modularity. It would be useful to know a much weaker but easily applicable condition than $\left(\xi^{\prime}\right)$ or $\left(\xi^{\prime \prime}\right)$ to serve along with $(\theta)$ as a set of necessary and sufficient conditions for modularity.

\section{Near-modular lattices.}

DEFinition 3. A finite lattice $L$ is near-modular, henceforth abbreviated NM, if and only if $(\theta)$ is valid and the Jordan-Dedekind chain condition is satisfied.

REMARK. It is conceivable that the JD chain condition is implied by $(\theta)$, though no proof was readily found. The imposition of the JD condition seems desirable, since it is satisfied in all finite semi-modular and modular lattices. Hence each element of a NM lattice $L$ will possess a uniquely determined rank.

THEOREM 4. In a NM lattice $L$ we have $D(x)=C(x)$ whenever $x$ is a point (atom) or dual point. Condition $\left(\xi^{\prime}\right)$ is satisfied by all pairs of points and $\left(\xi^{\prime \prime}\right)$ by all pairs of dual points.

Proof. Let $p$ be an arbitrary point of $L$ and $q$ any element in $C(p)$. By consideration of rank, $q$ is also a point which is distinct from $p$. Hence $q>q \cap p=z$, the zero element of $L$, and $q \in D(p)$. Thus $C(p)$ $\subseteq D(p)$. Equality of orders yields $C(p)=D(p)$. Any pair of points $p$ and $q$ cover their meet $z$ so that $q \in D(p)$. Hence $q \in C(p)$ so that $\left(\xi^{\prime}\right)$ is valid for $p$ and $q$. The remainder of Theorem 4 follows by duality.

Corollary. All NM lattices of rank less than 4 are modular.

TheOREM 5. There exist NM lattices of rank 4 that are nonmodular.

The smaller example $L_{4}$ of the two examples found was constructed from the finite projective geometry $\mathrm{PG}(2,2)$ as follows. If the points of $\mathrm{PG}(2,2)$ are designated by $1,2,3,4,5,6,7$, the lines, considered as sets of points, can be taken as $356,467,571,612,723,134,245$, and $u=$ 1234567. For $L_{4}$ take $u=1234567$, and the 7 dual points as 1247, 2351, $3462,4573,5614,6725,7136$, namely the complementary sets to the dual points of $\mathrm{PG}(2,2)$. The remaining elements of $L_{4}$ are generated by 
taking all point set meets of its dual points. The lines of $L_{4}$ are the $21=\left(\begin{array}{l}7 \\ 2\end{array}\right)$ pairs of points $12,13, \cdots, 67$; the points are $1,2,3,4,5,6,7$; and $z$ is the null set.

The automorphism group of $L_{4}$ is easily seen from the manner of its construction to be the same as that for $\operatorname{PG}(2,2)$, of order 168 . $L_{4}$ possesses dual automorphisms, one of which carries the planes in the order indicated above into the points $7,6,5,4,3,2,1$ respectively. Moreover, $L_{4}$ is a complemented point (atomic) lattice. It possesses no non-trivial homomorphic images, since all prime quotients are projective.

When the above procedure of construction of $L_{4}$ from $\mathrm{PG}(2,2)$ was applied to $\mathrm{PG}(2,3), \mathrm{PG}\left(2,2^{2}\right), \mathrm{PG}(3,2)$ and other $\mathrm{PG}\left(k, p^{n}\right)$, the lattices obtained were all found to violate $(\theta)$. Some of them violated also the JD chain condition.

The structure of $L_{4}$ suggested a method of obtaining additional examples of non-modular NM lattices as follows. Let $L_{t}$ consist of $z ; n$ points $p_{1}, p_{2}, \cdots, p_{n}$ where $n=1+\left(\begin{array}{l}t \\ 2\end{array}\right) ;\left(\begin{array}{l}n \\ 2\end{array}\right)$ lines consisting of all pairs of points : $p_{1} p_{2}, \cdots, p_{n-1} p_{n} ; n$ planes of which the first is the set of $t$ points $p_{i_{1}} p_{i_{2}} \cdots p_{i_{r}} \cdots p_{i_{t}}$ where $i_{r}=1+\left(\begin{array}{l}r \\ 2\end{array}\right),(r=1,2, \cdots, t)$ and the remaining planes are obtained from the first by repeated applications of the cyclic permutation $(123 \cdots n)$ to the subscripts; and $u=p_{1} p_{2} \cdots p_{n}$. This procedure yields for $t=1,2,3$ the Boolean algebras $B^{1}, B^{2}, B^{4}$ respectively. For $t=4$, the lattice $L_{4}$ described above is obtained. For $t=5$ a second example, $L_{5}$, of a non-modular NM lattice of length 4 is obtained. For $t \geqq 6$ one fails to obtain a lattice. It can readily be shown by consideration of certain congruences that for $t \geqq 6$ there always exist at least two pairs of planes, as described in the construction, which intersect in three or more points and other pairs of planes that intersect in less than two points. When two planes have three points $p$, $q, r$ in common, the lines $p q, p r$, and $q r$ have each of the planes as upper bound, but fail to have a least upper bound.

4. Extensions, In this section, methods of construction of other NM lattices from given ones are presented.

THEOREM 6. The direct product of NM lattices is also an NM lattice.

Proof. Let $L=L_{1} \times L_{2} \times \cdots \times L_{n}$ where the components are NM lattices. Represent each $a \in L$ in the usual way as the $n$-tuple $\left(a_{1}, \cdots\right.$, $\left.a_{n}\right)$ with $a \in L_{t}(i=1, \cdots, n)$, so that $a \cup b$ and $a \cap b$ are obtained by taking joins and meets respectively component-wise. Let $C(a)$ and $D(a)$ be the functions of Definition 2. Define $H(a)$ as the set of elements covering $a \in L$ and $K(a)$ as the set of elements covered by $a$. Let 
$C\left(a_{i}\right), D\left(a_{i}\right), H\left(a_{i}\right), K\left(a_{i}\right)$ be the corresponding sets with respect to $a_{i} \in L_{i}$. Now $a>b$ in $L$ if and only if $a_{j}>b_{j}$ for some $j$ and $a_{i}=b_{i}$ for $i \neq j$. It follows readily that

$$
\begin{aligned}
& N[C(a)]=\sum_{i=1}^{n} N\left[C\left(a_{i}\right)\right]+\sum_{i \neq j} N\left[H\left(a_{i}\right)\right] \cdot N\left[K\left(a_{j}\right)\right] \\
& N[D(a)]=\sum_{i=1}^{n} N\left[D\left(a_{i}\right)\right]+\sum_{i \neq j} N\left[K\left(a_{j}\right)\right] \cdot N\left[H\left(a_{i}\right)\right]
\end{aligned}
$$

The last summations of the two equations are equal. By hypothesis $\left(\theta_{i}\right): N\left[C\left(a_{i}\right)\right]=N\left[D\left(a_{i}\right)\right]$ for $i=1, \cdots, n$. Hence $(\theta)$ is valid in $L$.

Notation. $L=a / b$ indicates a lattice with unit element $a$ and zero element $b$. The set sum and product of lattices $L_{1}$ and $L_{2}$, considered only as sets of elements, will be denoted by $L_{1}+L_{2}$ and $L_{1} \cdot L_{2}$ respectively.

Lemma 1. If $L_{1}=a_{1} / z$ possesses $a$ dual ideal $a_{1} / b_{1}$ isomorphic to an ideal $a_{2} / b_{2}$ of $a$ second lattice $L_{2}=u / b_{2}$, then by identifying as $x$ each pair of elements $x_{1} \in a_{1} / b_{1}$ and $x_{2} \in a_{2} / b_{2}$ that correspond under the isomorphism, a lattice $L=u / z$ can be constructed having $L_{1}$ as an ideal and $L_{2}$ as a dual ideal such that $L=L_{1}+L_{2}$ and $a / b \equiv L_{0}=L_{1} \cdot L_{2}$.

The elements of $L$ are taken as the identified elements $x \in a / b$ and the remaining elements of $L_{1}$ and $L_{2}$. Join $U$ and meet $\cap$ in $L$ are defined in terms of $U_{1}, \cap_{1}$ in $L_{1}$ and $U_{2}, \cap_{2}$ in $L_{2}$ according to the cases :

$$
\begin{aligned}
& \left.\begin{array}{l}
r \cup s=r \cup_{i} s \\
r \cap s=r \cap_{i} s
\end{array}\right\} \quad r, s \in L_{i}, \quad(i=1,2) \\
& \left.\begin{array}{l}
r \cup s=s \cup r=\left(r \cup_{1} b\right) \cup_{2} s \\
r \cap s=s \cap r=r \cap_{1}\left(a \cap_{2} s\right)
\end{array}\right\} \quad r \in L_{1}, s \in L_{2} .
\end{aligned}
$$

The verification of the lattice postulate is routine and is omitted. This method of extension was first employed systematically by M. Hall and R. P. Dilworth [2; Lemma 4.1].

In Lemmas 2, 3, 4 and Theorem 7 following, let $L=u / z$, ideal $L_{1}=$ $a / z$, dual ideal $L_{2}=u / b$, and quotient sublattice $L_{0}=a / b$ be related as in Lemma 1: $L=L_{1}+L_{2}$ and $L_{0}=L_{1} \cdot L_{2}$. We note that $L-L_{2}, L_{0}, L-L_{1}$ is a partitioning of $L$ into disjoint subsets.

Lemma 2. If $s>r$ in $L$, then $s$ and $r$ are both in $L_{1}$ or both in $L_{2}$.

Proof. Obviously impossible is the case $s \notin L_{2}, r \notin L_{1}$. Assume that $s \notin L_{1}, r \notin L_{2}$; that is, $b \leqq s \nsubseteq a, a \supseteqq r \nsupseteq b$. Then $s=s \cup r=s \cup(b \cup r) \supset b \cup r$, otherwise $s \subseteq b \cup r \subseteq a$, a contradiction. Furthermore $b \cup r \supset r$, otherwise $b \subseteq r$, 
a contradiction. Thus the covering $s>r$ is violated and the only possible cases are as stated.

Lemma 3. If $D_{i}(x), C_{i}(x)$ are the functions of Definition 2 relative to $L_{i}(i=0,1,2)$, then

(3) $D_{0}(x)=D_{1}(x) \cdot D_{2}(x)$

(3') $C_{0}(x)=C_{1}(x) \cdot C_{2}(x)$

(4) $D(x)=D_{1}(x)+D_{2}(x)$

(4') $C(x)=C_{1}(x)+C_{2}(x)$

Proof. (3) holds since $r \in D_{0}(x): r>r \cap x<x$ with $r, r \cap x, x$ all in $L_{0}=L_{1} \cdot L_{2}$ if and only if $r>r \cap x<x$ with $r, r \cap x, x$ all in both $L_{1}$ and $L_{2}$; that is, $r \in D_{1}(x), D_{2}(x), D_{1}(x) \cdot D_{2}(x)$. Next, $r \in D_{1}(x): r>r \cap x<x$ with $r, r \cap x, x$ all in $L_{1}$ implies $r \in D(x): r>r \cap x<x$ with $r, r \cap x, x$ all in $L$. Thus $D_{1}(x) \subseteq D(x)$. Similarly $D_{2}(x) \subseteq D(x)$ so that $D_{1}(x)+D_{2}(x) \subseteq D(x)$. For demonstration of the less trivial reverse inclusion let $r \in D(x): r>r \cap x$ $<x$ in $L$. If $r \cap x \notin L_{2}$, both $r$ and $x$ are in $L_{1}$, along with $r \cap x_{1}$, by Lemma 2. For this case $r \in D_{1}(x)$. If $r \cap x \in L_{2}$, then certainly also are both $r$ and $x$. Whence $r \in D_{2}(x)$. We thus obtain $D(x) \subseteq D_{1}(x)+D_{2}(x)$ and therefore (4). Dually $\left(3^{\prime}\right)$ and $\left(4^{\prime}\right)$ are valid.

LEMma 4. The following statements of orders are valid:

$$
\begin{aligned}
& N[D(x)]+N\left[D_{0}(x)\right]=N\left[D_{1}(x)\right]+N\left[D_{2}(x)\right] \\
& N[C(x)]+N\left[C_{0}(x)\right]=N\left[C_{1}(x)\right]+N\left[C_{2}(x)\right]
\end{aligned}
$$

This follows immediately from Lemma 3.

THEOREM 7. If any three of $L, L_{1}, L_{2}, L_{0}$, related as in Lemmas 1-4, are near-modular, then all are near-modular.

Proof. Equality of three pairs of corresponding members of (5) and $\left(5^{\prime}\right)$ implies equality of the remaining pair.

REMARKS. It is no doubt possible to construct non-modular NM lattices in other ways; for example, by piecing together several NM lattices to become the ideals of $L$ and several others to become the dual ideals of $L$. Such a construction would require perhaps a more precise knowledge of the basic structure of a NM lattice.

A sublattice, and even a quotient sublattice, of a NM lattice is not necessarily near-modular. It is an open question whether or not the homomorphic image of a NM lattice is near-modular. 


\section{REFERENCES}

1. G. Birkhoff, Lattice theory, rev. ed., Amer. Math. Soc. Colloquium Publications, New York, 1948.

2. M. Hall and R. P. Dilworth, The imbedding theorem for modular lattices, Ann. of Math., 45 (1944), 450-456.

UNIVERSITY OF WASHINGTON 


\section{PACIFIC JOURNAL OF MATHEMATICS}

\section{EDITORS}

David Gilbarg

Stanford University

Stanford, California

R. A. Beaumont

University of Washington

Seattle 5 , Washington
A. L. Whiteman

University of Southern California Los Angeles 7, California

E. G. Straus

University of California

Los Angeles 24, California

\section{ASSOCIATE EDITORS}
E. F. BECKENBACH
C. E. BURGESS
M. HALL
E. HEWITT
A. HORN
V. GANAPATHY IYER
R. D. JAMES
M. S. KNEBELMAN

L. NACHBIN

I. NIVEN

T. G. OSTROM

H. L. ROYDEN
M. M. SCHIFFEI

G. SZEKERES

F. WOLF

K. YOSIDA

\section{SUPPORTING INSTITUTIONS}

UNIVERSITY OF BRITISH COLUMBIA

CALIFORNIA INSTITUTE OF TECHNOLOGY

UNIVERSITY OF CALIFORNIA

MONTANA STATE UNIVERSITY

UNIVERSITY OF NEVADA

OREGON STATE COLLEGE

UNIVERSITY OF OREGON

OSAKA UNIVERSITY

UNIVERSITY OF SOUTHERN CALIFORNIA
STANFORD UNIVERSITY

UNIVERSITY OF TOKYO

UNIVERSITY OF UTAH

WASHINGTON STATE COLLEGE

UNIVERSITY OF WASHINGTON

AMERICAN MATHEMATICAL SOCIETY

CALIFORNIA RESEARCH CORPORATION

HUGHES AIRCRAFT COMPANY

THE RAMO-WOOLDRIDGE CORPORATION

Printed in Japan by Kokusai Bunken Insatsusha

(International Academic Printing Co., Ltd.), Tokyo, Japan 


\section{Pacific Journal of Mathematics}

\section{Vol. 8, No. 1 \\ March, 1958}

Shimshon A. Amitsur, Commutative linear differential operators ......... 1

Masahiko Atsuji, Uniform continuity of continuous functions of metric

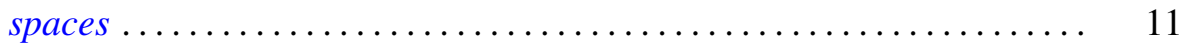

S. P. Avann, A numerical condition for modularity of a lattice . . . . . . . . . 17

Raymond G. D. Ayoub, A mean value theorem for quadratic fields........ 23

Errett Albert Bishop, Subalgebras of functions on a Riemann surface ..... . 29

Shaul Foguel, The relations between a spectral operator and its scalar

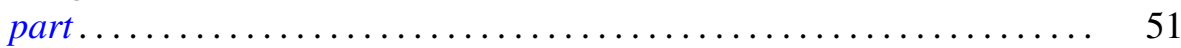

John Rolfe Isbell, Euclidean and weak uniformities ................. 67

Samuel Karlin and James L. McGregor, Many server queueing processes with Poisson input and exponential service times .............. 87

Paul Joseph Kelly and Ernst Gabor Straus, Curvature in Hilbert geometries....................................... 119

John W. Lamperti, Stationary measures for certain stochastic processes . . . 127

Richard Scott Pierce, Distributivity and the normal completion of Boolean

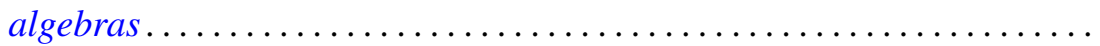

F. M. Ragab, Transcendental addition theorems for the hypergeometric

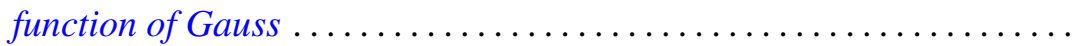

William T. Reid, Principal solutions of non-oscillatory self-adjoint linear differential systems ................................ 147

Maurice Sion, On general minimax theorems .................... 171

Chien Wenjen, On semi-normed ${ }^{*}$-algebras .................... 177 